\title{
Large Area Sea Mapping with Ground-Ionosphere-Ocean-Space (GIOS)
}

\section{Paul A. Bernhardt ${ }^{* 1}$, Stanley J. Briczinski', Carl L. Siefring ${ }^{1}$, Donald E, Barrick ${ }^{2}$, Jehu Bryant ${ }^{3}$, Andrew Howarth ${ }^{4}$, Gordon James ${ }^{4}$, Greg Enno ${ }^{4}$, Andrew $\mathrm{Yau}^{4}$}

\author{
${ }^{1}$ Plasma Physics Division, Naval Research Laboratory, Washington DC, 20375, USA. \\ (E-mail: paul.bernhardt@nrl.navy.mil), ${ }^{2}$ CODAR Ocean Systems, 1914 Plymouth Street, Mountain View, \\ California 94043 USA, ${ }^{3}$ Raytheon IIS, Chesapeake, VA, ${ }^{4}$ University of Calgary, Department of Physics \\ and Astronomy, Institute for Space Research Calgary, AB, T2N 1N4, CANADA
}

\section{Introduction}

Knowledge of the sea is of primary importance for both ship transportation and HF radar clutter prediction. Remote measurements of sea state provide data inputs to operational models for maritime domain awareness. Ocean measurement techniques employing radio waves include (1) passive microwave imagery, (2) ocean microwave emissivity for surface waves, foam detection, temperature and salinity, and (3) microwave and HF radars for wind speed and direction. The HF radars detect ground surface waves over a limited $(200 \mathrm{~km})$ range for backscatter measurements of wind speed and ocean currents. Space-based ocean sensors include microwave scatterometers, altimeters for topography, GPS/GNSS satellite receivers for reflectometry, and imaging radars (SAR) [1]. These existing measurement techniques can be complemented and enhanced using a new technique with ground HF transmissions scattering off large areas of the ocean to satellites in low earth orbit for broad area mapping of the ocean surface properties.

\section{HF GIOS Concept}

A new concept has been developed called HF Ground-Ionosphere-Ocean-Space (GIOS) which can view vast regions of the Earth's surface (Figure 1). Ground HF transmissions are reflected by the ionosphere to illuminate the ocean over a few thousand kilometers. HF receivers on low-earth-orbit satellites detect the radio waves scattered by the sea and land surface. Using the theory of radio wave scatter from ocean surfaces [2], the GIOS data is then processed to yield the directional wave-height spectrum of the ocean. The GIOS technique has several advantages over existing remote sensing methods. First, a large area of the ocean can be sampled to yield the wave-height characteristics with high, km-scale resolution. This measurement scale matches the grid size used in physics-based oceanographic models. The wave height spectrum can be directly compared with temporal frequency spectrum [3] obtained with buoys at specific points in the ocean volume. Furthermore, the GIOS technique uses HF waves which penetrate the dense rain found in hurricanes. Microwave attenuation inside strong sea storms blocks mapping of the sea surface.

The details on the propagation of an HF wave from a ground transmitter to a satellite in low-earth orbit are illustrated in Figure 1. A linearly polarized antenna on the earth's surface will excite an electromagnetic (EM) wave which will decompose into an ordinary mode (Blue) and extraordinary mode (Purple) upon entering bottomside of the ionospheric plasma layer (green). Each mode has a characteristic right and left hand circular polarization which can be isolated using a crossed-dipole receiver antenna. Waves with elevations below a critical angle for each polarization will be refracted by the ionosphere to the ground for illumination of the ocean or land surface. The skip zone is defined by the shortest ray distance for each mode that hits the earth. An enhancement in the intensity of the HF signal on the earth is found at the 
skip distance caustic where rays are closest together. The scatter from the ocean surface is either by a coherent (mirror) or an incoherent (rough) process. For coherent specular scatter, the incident and scatter angles are identical. For incoherent scatter, the scatter angle can occur at any value and the amplitude of the scattered wave is determined by the amplitude of the incident wave, the Fresnel Zone area of the scatter surface, and the properties of the irregular surface. The scattered HF wave propagates on ray paths are bent by the refractive index ionosphere before being received on the satellite. The group delay between the transmitter and satellite represents the time between a transmitted pulse propagation along the GIOS path. The Doppler frequency shift is associated the incident wave angle on the satellite projected onto the satellite velocity vector. The satellite data are processed to give the signal amplitude at each group delay and Doppler frequency to be yield the wave-height spectral component at each point on the ocean. This geometry provides bistatic remote sensing of the ocean by taking advantage of the satellite motion along its orbit.

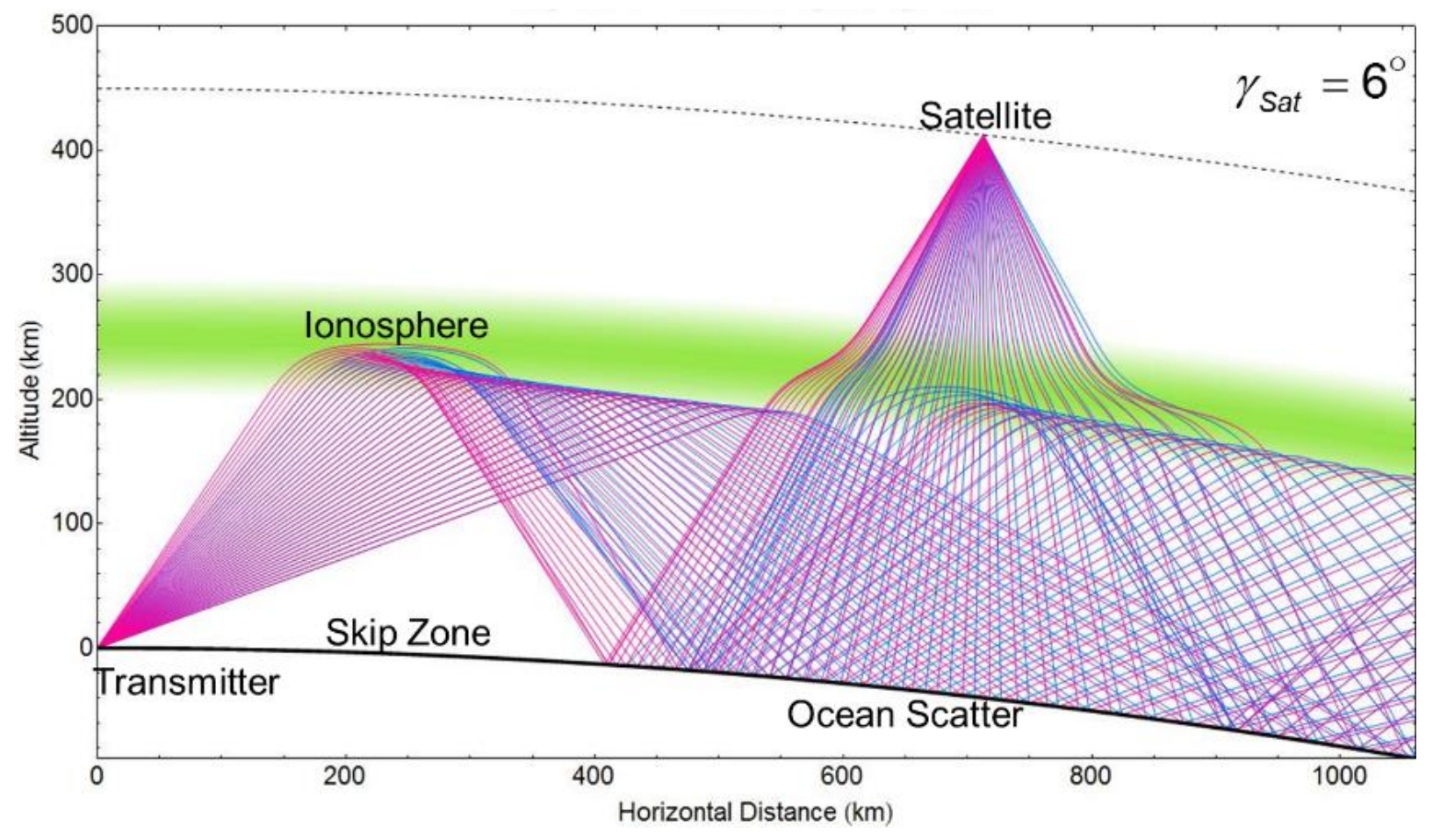

Figure 1 Ground-Ionosphere-Ocean-Space (GIOS) concept with sky wave illumination of the ocean for large area mapping of surface roughness. The satellite receiver determines the position of the ocean scatter by time delay (group range) and frequency (satellite motion Doppler shift). Ray trajectories are shown for a $17.5 \mathrm{MHz}$ transmissions passing through an ionosphere with a 10 $\mathrm{MHz}$ critical frequency. The O-Mode (Blue) and X-Mode (Purple) rays propagate along different paths to the ocean and satellite. Crossed dipoles on the satellite are used to separate the polarization modes. At the point in the simulated propagation study, the satellite makes a geocentric angle of 6 degrees relative to the transmitter location.

The largest HF scatter signal is provided by coherent scatter where the ocean acts like a mirror $[4,5]$. The specular reflection of a circularly polarized wave incident on a highly conducting ocean 
changes the sense of polarization (left to right and right to left) causing mode conversion. The coherent scatter reflection coefficient from fresh water is up to $50 \%$ of the value for scatter from sea water. Also, a layer of sea ice influences the reflection of HF waves. Thus, the amplitude of the coherent HF wave scatter wave can provide measurements of both salinity and sea ice thickness.

The simplest description of incoherent HF wave scatter from the ocean involves an isotropic waveheight spectrum $\mathrm{S}_{\mathrm{k}}(\mathrm{k})$ [2]. The ocean waveheight spectrum relates the statistics of their vertical displacement to their temporal period $(T)$ or their length $(\lambda)$. The temporal spectrum is a function of wave frequency $\omega=2 \pi / \mathrm{T}$ and the spatial spectrum is written in terms of wave-number $\mathrm{k}=2 \pi / \lambda$. The temporal and spatial waveheight spectra for wind driven waves are related by the dispersion equation for deep water gravity waves $\omega^{2}=\mathrm{kg}$. Figure 2 shows a representative wave height spectrum derived from the Fourier Transform of temporal variations in wave height what were measured on a buoy. The temporal spectrum was converted to wave number using the dispersion equation and fit with the Pierson Moskowitz functional form given by

$$
S_{\omega}(\omega)=\alpha \frac{g^{2}}{\omega^{5}} e^{-\frac{5}{4}\left(\frac{\beta g}{U \omega}\right)^{4}}\left(m^{2}-s\right) \text { or } S_{k}\left(k_{r}\right)=\frac{g^{2}}{2 \pi \omega^{3}} S_{\omega}\left(\omega=\sqrt{k_{r} g}\right)=\frac{\alpha}{2 \pi k_{r}^{4}} e^{-\frac{5}{4} \frac{\beta^{4} g^{2}}{4 U^{4} k^{2}}}\left(m^{4}\right)
$$

This spectrum is characterized by a maximum near wave numbers of $0.025 \mathrm{~m}^{-1}$ representing swell dimensions around 100 meters. The incident HF wave samples the waveheight spectrum at different wave numbers depending on the direction of the scatter angle. For a surface wave (90 degree incidence), backscatter (90 degree scatter angle) occurs for wave numbers well above the peak location. Oblique forward scatter near -45 degree scatter angle samples the waveheight spectrum at its peak.

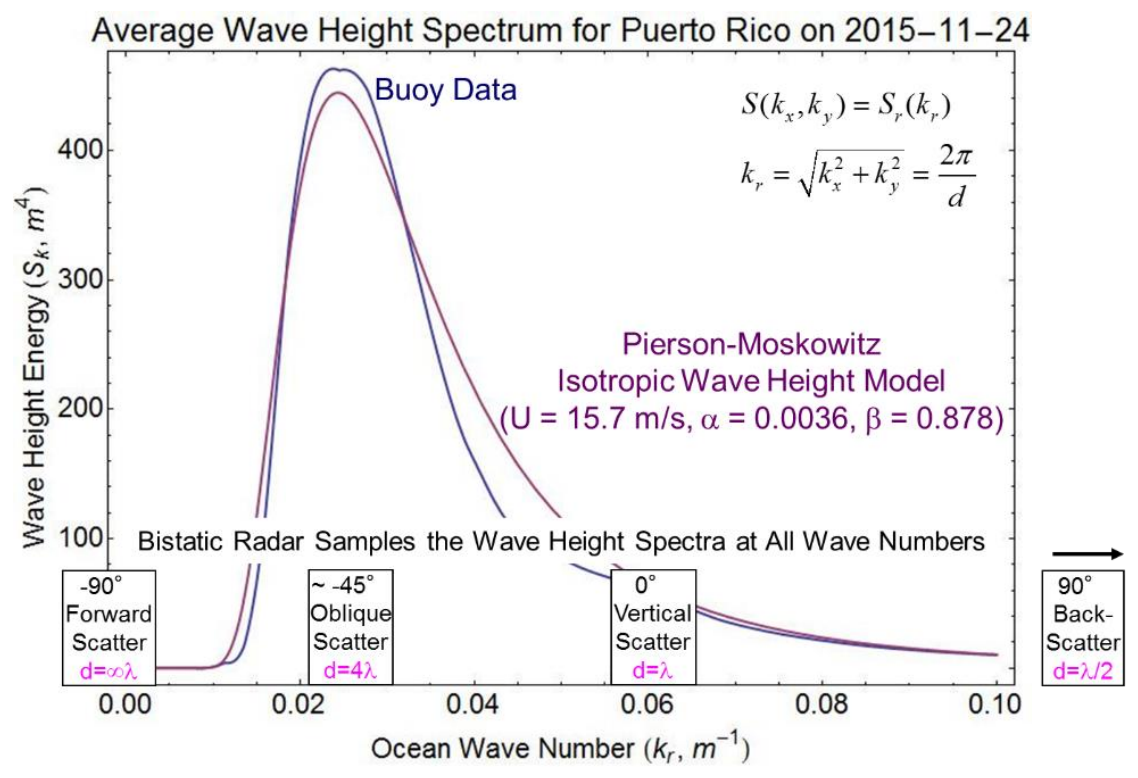

Figure 2. Example of a wave height wave number spectrum derived from Puerto Rico wave buoy data (blue) fit with the Pierson-Moskowitz model using a wind speed of 15.7 $\mathrm{m} / \mathrm{s}$.

A model simulation that includes both coherent and incoherent scatter from sea water with swells driven by a neutral wind has been used to derive the scatter shown in Figure 3. The coherent scatter is about $30 \mathrm{~dB}$ larger than the incoherent scatter for the $17.5 \mathrm{MHz}$ EM frequency used in the model. The incoherent scatter cross section is directly related to the waveheight spectra by 


$$
\sigma_{L R}=4 \pi k_{0}^{4}\left|\alpha_{L R}^{\prime}\right|^{2} S_{k}\left[k_{0} \sqrt{\left(\sin \theta_{s} \cos \phi_{s}-\sin \theta_{i}\right)^{2}+\left(\sin \theta_{s} \sin \phi_{s}\right)^{2}}\right]
$$

where $\mathrm{k}_{0}=$ is the wave number of the transmitter electromagnetic wave, $\alpha^{\prime}{ }_{\mathrm{LR}}$ is the polarization coupling scatter coefficient, and $\theta_{\mathrm{i}}, \theta_{\mathrm{s}}$, and $\phi_{\mathrm{s}}$ are the incident angle, scatter angle, and scatter azimuths, respectively $[4,6]$. The incoherent modes are scattered above and below the coherent modes. The reception on a satellite depend on which of the ocean to space ray paths can penetrate to the ionosphere. Interpretation of the received amplitudes and polarizations to yield ocean surface parameters depends on a number of factors including (1) amplitude reductions from ray path spreading for propagation from the ground-ionosphere-ocean segment, Fresnel Zone contributions at the reflection plane [5], coherent and incoherent scatter, ray path spreading for propagation from the ocean through the ionosphere to the satellite, and plasma refractive index effects on impedance of the satellite antenna in the ionosphere.

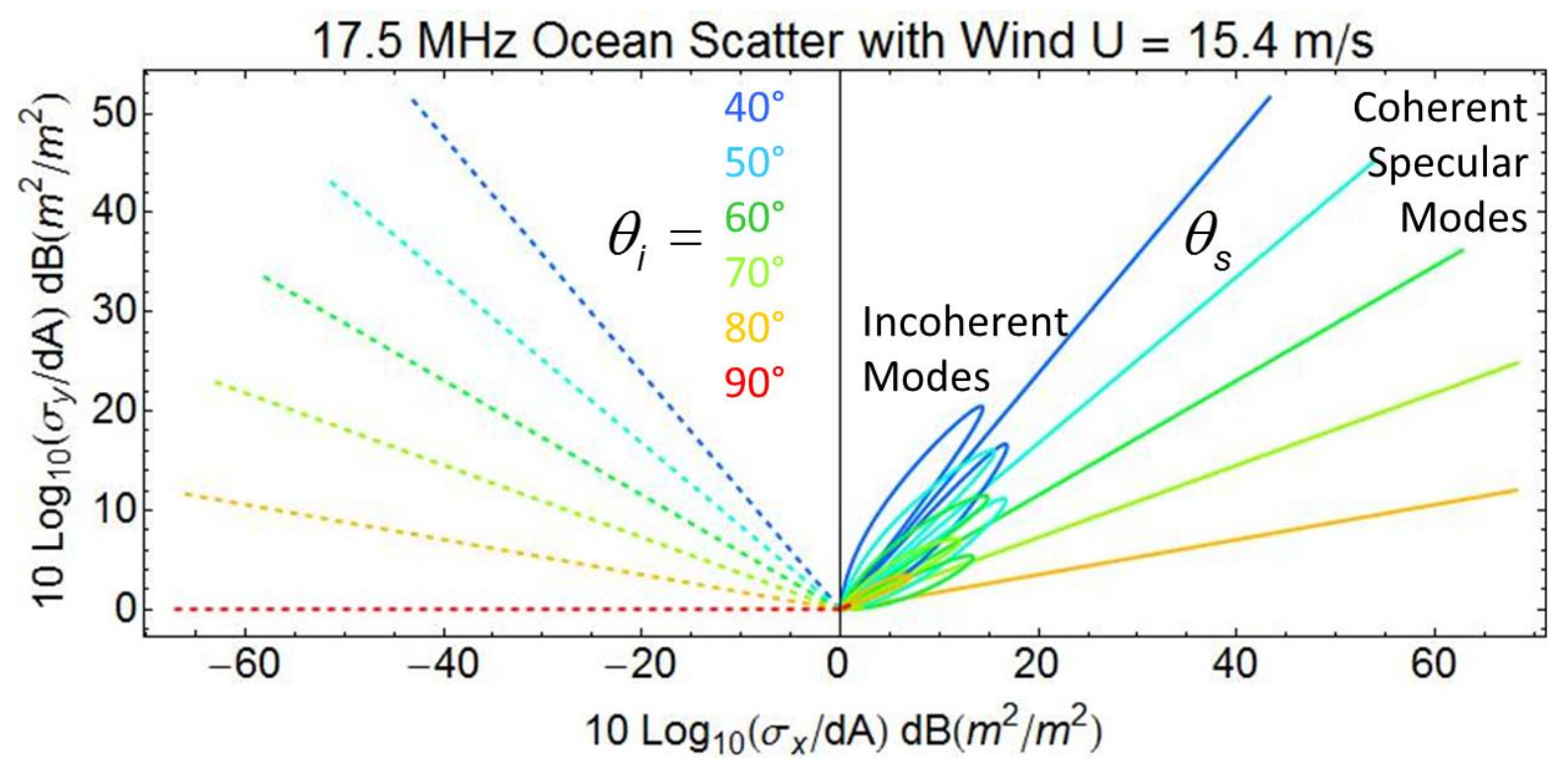

Figure 3. Scatter modes for HF waves incident on the ocean surface after ionospheric reflection. The incidence angles vary from 40 to 90 degrees to give both specular (coherent mode) and nonspecular (incoherent mode) reflections. The amplitude specular reflections yield both ocean salinity and can detect sea ice layers. The amplitude of the non-specular reflections are directly related to the wave height spectrum.

\section{HF GIOS Testing with Existing Ground and Space Systems}

The GIOS program at NRL is being developed using both experimental and theoretical methods. To test the GIOS concept, ground HF transmissions from over-the-horizon radars were employed to scatter sky wave signals from the ocean to radio receivers in low-earth-orbit. The HF receiver (RRI) on the Canadian ePOP/CASSIOPE satellite [7] has collected radio signals scattered from the ocean illuminated by ground transmitters in the US, Australia and Northern Europe. This satellite has two dipole antennas in a crossed configuration (Figure 4) to measure HF waves below $18 \mathrm{MHz}$. Right and left hand circular polarization is synthesized from the data from the in phase (I) and quadrature (Q) data provided by the RRI digital instrument. 


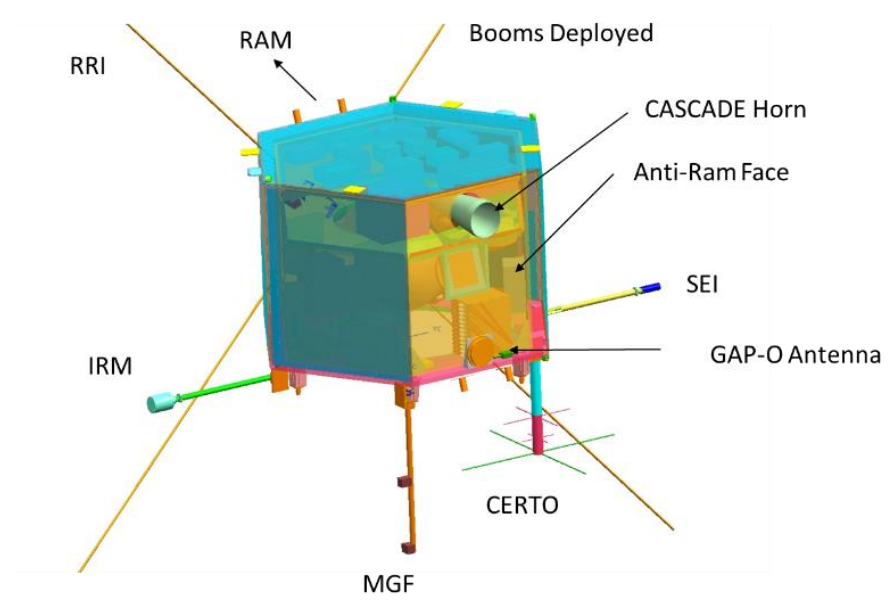

Figure 4. CASSIOPE satellite with the ePOP payload including the radio receiver instrument (RRI) for ocean observations and other plasma environment diagnostics. The ePOP RRI covers DC to $18 \mathrm{MHz}$ using 6-m crossed-dipole antennas. The other ePOP instruments measure in situ ion and electron densities, ionospheric profiles, neutral densities, and magnetic fields and are supported by data telemetry downlinks.

For the ground HF transmission source, the Relocatable Over the Horizon Radar (ROTHR) system in Chesapeake Virginia was used to illuminate the ocean extending from coast of Florida to south of Jamaica (Figure 4). The ePOP satellite orbit along close to this illumination path recorded the HF radar transmissions at $17.5 \mathrm{MHz}$. An FMCW waveform with $8 \mathrm{kHz}$ bandwidth and $6.25 \mathrm{~Hz}$ repetition frequency was scattered by the ocean.

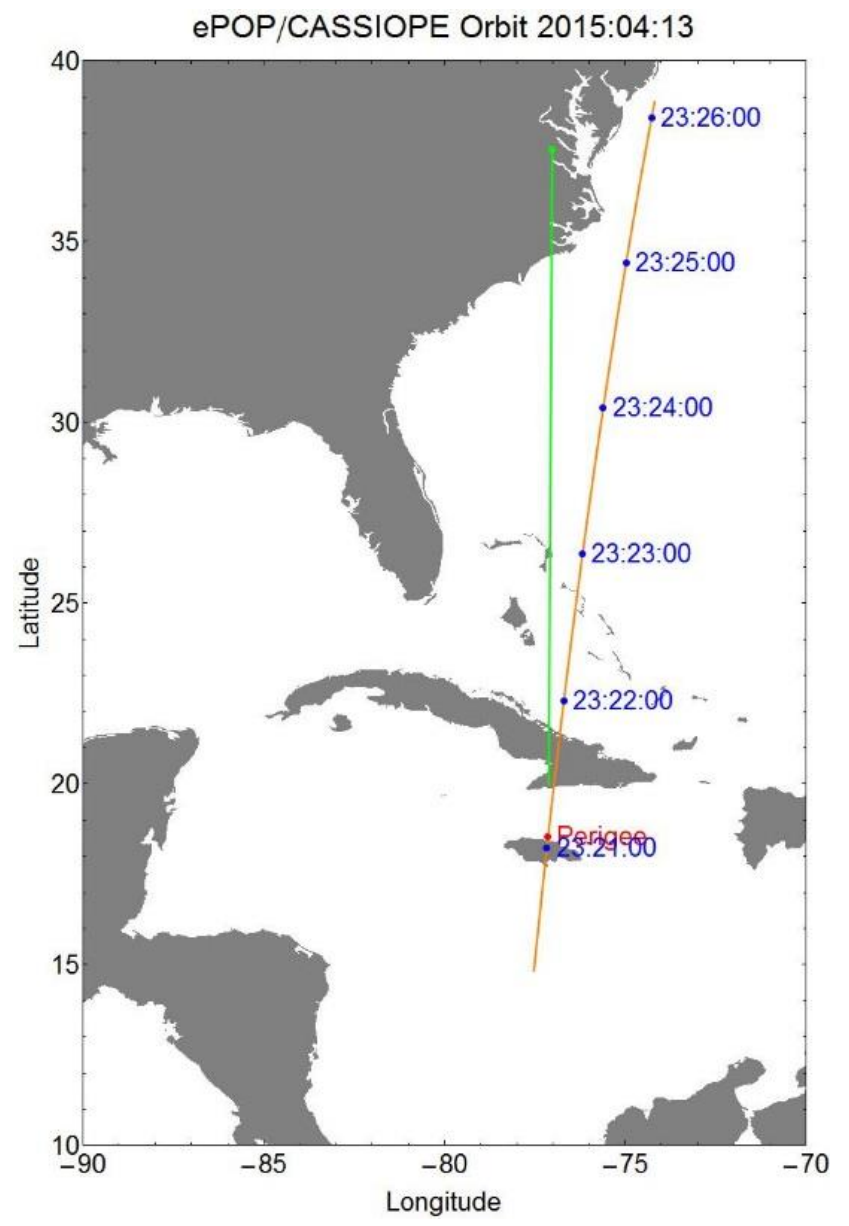

Figure 5. Ocean and island illumination by an 8 degree $\mathrm{HF}$ radar beam (green) at $17.5 \mathrm{MHz}$ (Left). The HF receiver on the ePOP satellite is observing the ocean along a nearly parallel orbit (orange). The HF radar beam illuminates the Caribbean Sea as well as Cuba and Jamaica. Shallow regions to the north of Cuba introduce wave height growth (shoaling) that is detected by increased radio wave scatter from this region. The perigee of the ePOP orbit is over Jamaica at an altitude of $320 \mathrm{~km}$. This altitude is close to the peak height of the ionosphere. 
Range processing of the GIOS data shows features of the ocean associated with winddriven swells, coastal shoaling, and dry land (Figure 6). When the satellite is near the latitude of the transmitter, (1) a direct path is detected with large amplitude and minimum group range and (2) the weak ocean scatter comes from the region past the skip zone for a large group range. The boundary of the skip zone is a large amplitude region associated with the skip distance caustic shown in Figure 1. When the satellite is south of the transmitter, the dire ct path group range increases and the ocean scatter group range decreases with a minimum range directly over the skip zone boundary.

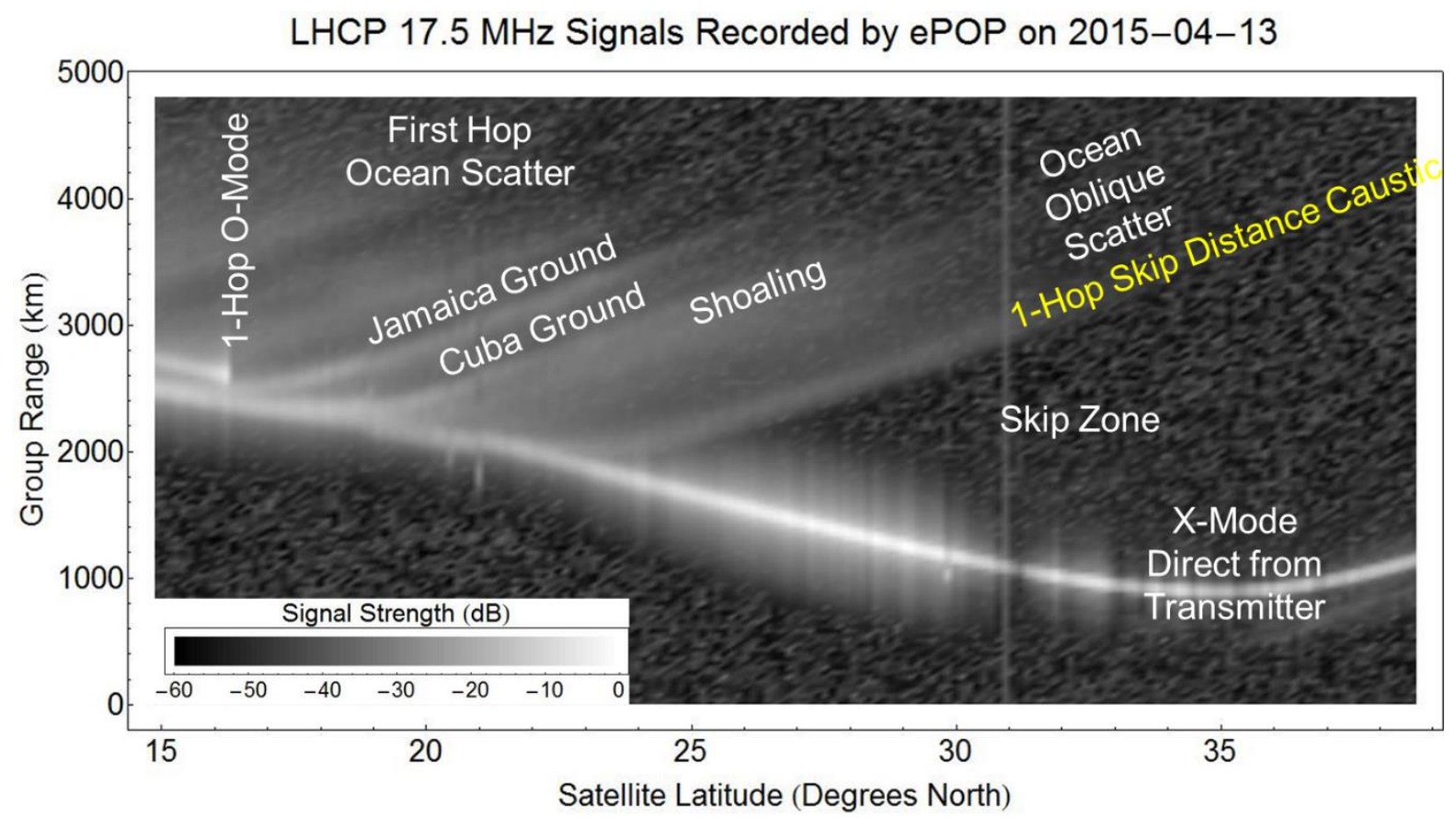

Figure 6. Surface scatter of HF waves to the ePOP radio receiver instrument (RRI) at 320 to 370 $\mathrm{km}$ altitude showing multiple features of the ionosphere, ocean and shore. Range and Doppler processing of the transmitted HF waveforms can yield maps of the wave-height spatial spectrum along the earth illuminated by the HF beam. The HF transmitter system at Chesapeake Virginia radiated a narrow ( 8 degree) beam at $17.5 \mathrm{MHz}$ that illuminated the Caribbean Sea as well as Cuba and Jamaica for the orbit track of Figure 5. The HF receiver on the ePOP satellite observed the ocean along an orbit nearly parallel to this HF radio beam. Shallow regions to the north of Cuba introduce wave height growth (shoaling) that is detected by increased radio wave scatter from this region.

Range and Doppler processing of the radar waveforms yields an ocean scatter map at each time in the ePOP orbit. When the satellite is close to the ground point of the transmitter, both the direct signal and the quasi-backscatter from the ocean past the skip distance reaches the satellite (Figure 7). The narrow beam of the HF radar illuminates a ground region extending nearly along the satellite orbit line (Figure 7, top). A "swoosh" is formed in the weak ocean scatter because of coupling between the propagation distance and HF the wave vector angle relative to the satellite velocity vector. The ocean scatter is very weak because it is far from the optimum forward scatter geometry illustrated by Figure 3. 


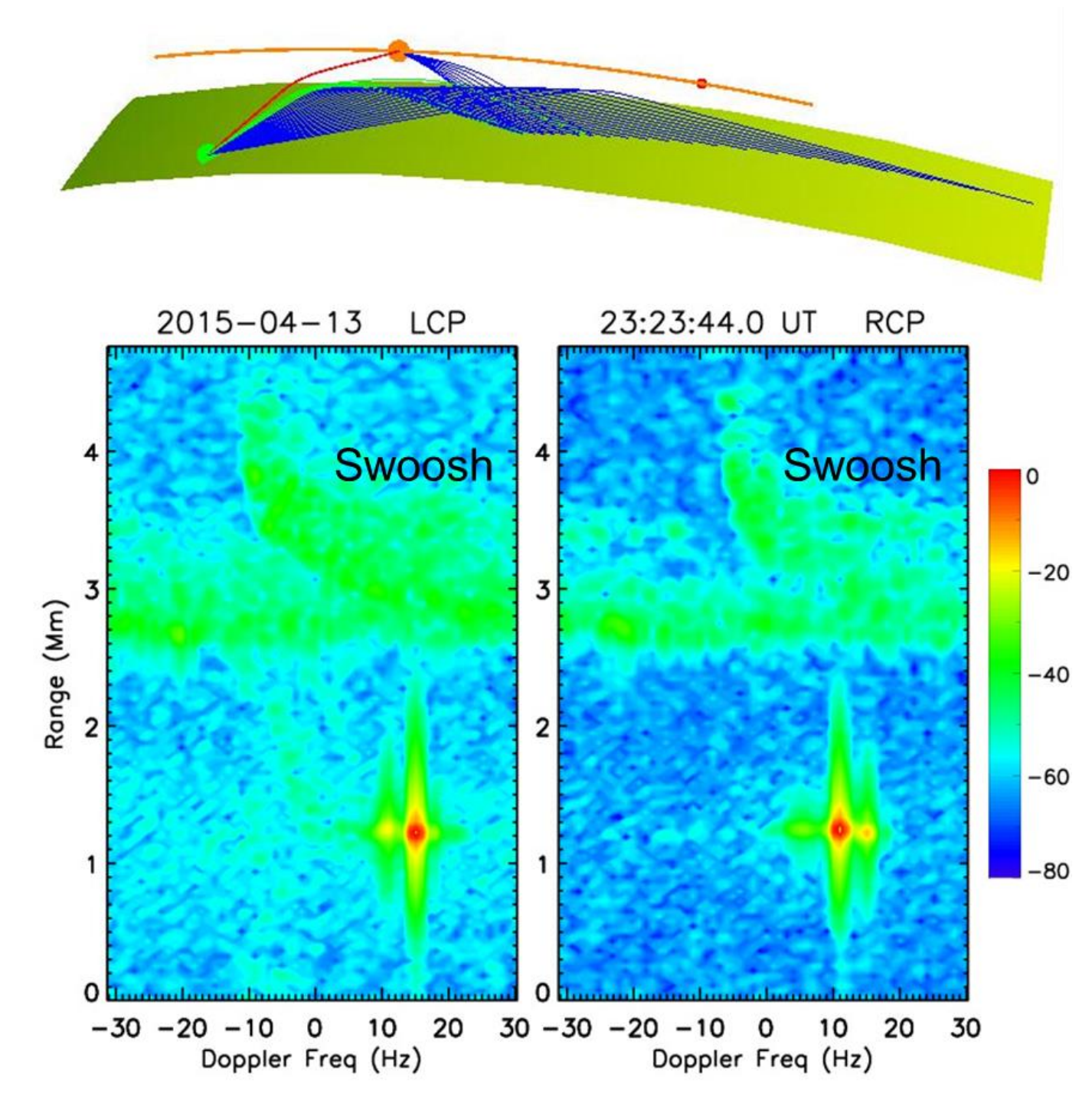

Figure 7. Satellite measurements of ocean scatter with a position relative to ocean illumination in a quasi-backscatter geometry. The geometry schematic (top) shows both direct and backscatter paths for the HF measurements. The range-Doppler display (bottom) shows a strong direct path and a Doppler spread "swoosh" signature that is $40 \mathrm{~dB}$ weaker than the direct wave. Increasing range is associated with negative Doppler in the satellite from the satellite motion. The range width at a fixed Doppler frequency is a result of out of plane ocean scatter.

When the satellite receiver is well past the HF skip zone, both forward and backscatter from the ocean can be obtained in the plane of the satellite orbit (Figure 8). The direct path from the transmitter is screened out by the ionosphere and only ocean scatter signals are recorded at the spacecraft (Figure 8, top). Propagation through the ionosphere distorts the HF ray paths making them nearly horizontal at the ends of the scatter ray paths and nearly vertical from the sub-satellite point on the earth. Thus there will be a wide range of Doppler frequency shifts with positive Doppler (for a northward moving satellite) for the forward scattered rays and a negative Doppler for the backscattered rays. This same rays will have corresponding small and large group paths.

Using the same single scale as Figure 7, the range-Doppler measurements of ocean scatter 
when the satellite is well past the skip zone shows as strong signal associated with the minimum range (Figure 8, bottom). To interpret this feature to yield the wave height spectrum from the ocean scatter coefficient defined by (2), the propagation distortions produced by the refraction of the ionosphere have to be removed. This requires knowledge of the F-layer ionosphere profile over the ocean scatter region.

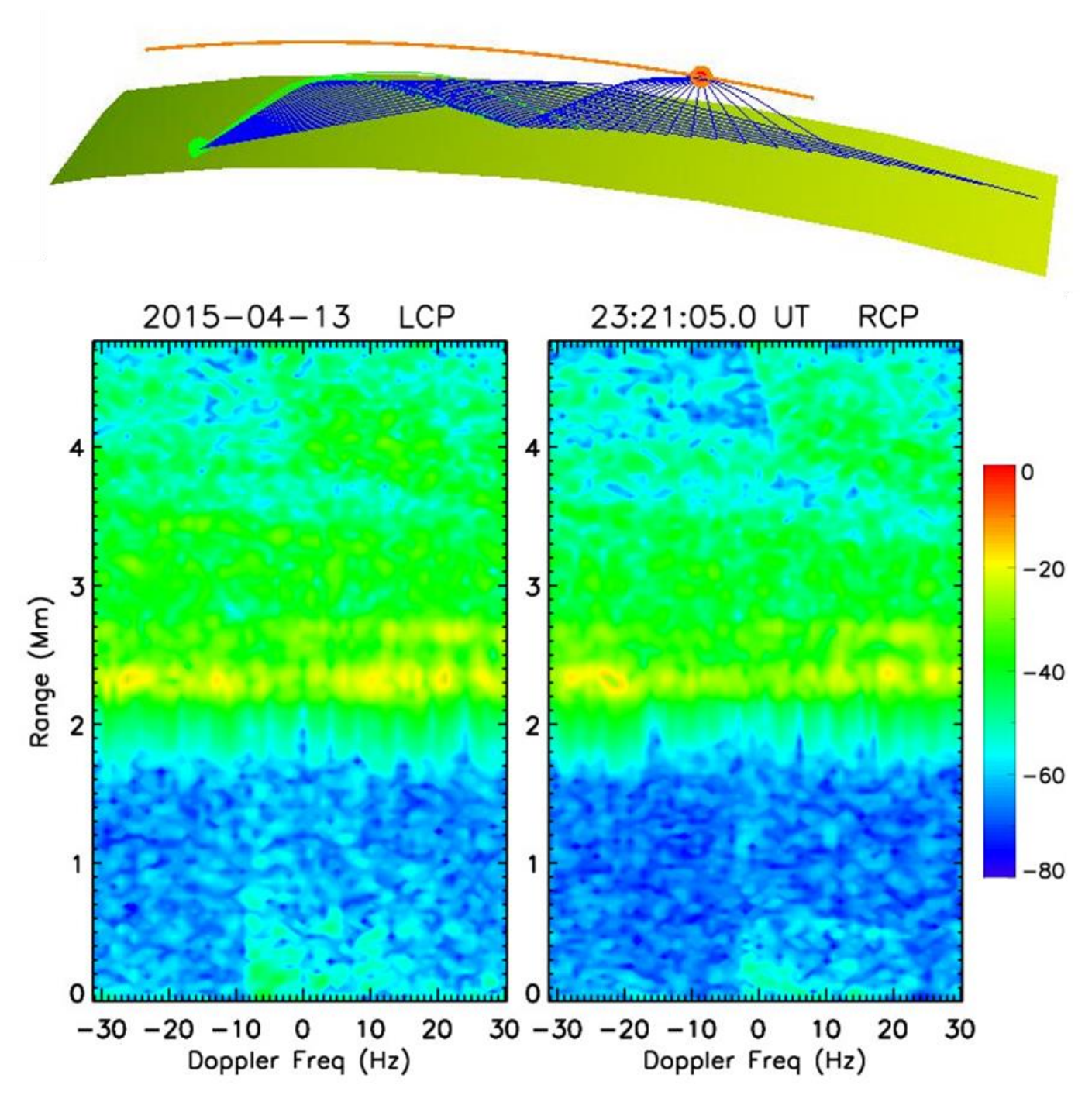

Figure 8. Satellite measurements of ocean scatter with a position relative to ocean illumination in a quasi-forward-scatter geometry. The geometry schematic (top) shows a wide range of ocean scatter paths that arrive at the satellite. These paths sample the waveheight spectrum at a wide range of wave numbers. The range-Doppler display (bottom) shows a peak in the backscatter for a minimum range near $2300 \mathrm{~km}$. This corresponds to the peak in the wave height spectrum illustrated in Figure 2. The Doppler spectrum is wrapped with an ambiguity of $62.5 \mathrm{~Hz}$ so the actual Doppler spread at the satellite will extend from -200 to $200 \mathrm{~Hz}$. The goal of the GIOS program is to use the range and Doppler processing of the transmitted HF waveforms to yield maps of the wave-height spatial spectrum along the earth illuminated by the HF beam.

The ePOP data are being interpreted using a GIOS model developed at NRL that describes 
the HF propagation through the ionosphere and the scatter from the ocean. The linear polarization of the transmitted signal is decomposed into right and left hand circular polarizations that propagate with different group velocities as the wave is reflected and refracted by the bottomside ionosphere. After reaching the ocean, the wave is scattered along non-specular paths to the satellite which will eventually enable a measurement of the full wavenumber range of the wave height spectrum giving estimates in the open ocean regions.

The primary limitation for the wave height spectrum determination is the F-layer ionosphere which peaks at about $300 \mathrm{~km}$ altitude. Future ePOP measurements will made when the ionospheric peak height is greater than the perigee altitude of the satellite. What is needed for the GIOS program is a satellite that flies below the ionosphere. To eliminate the ocean to satellite distortions of the ionosphere, NRL has designed a new satellite called CARINA to fly around 200 to $250 \mathrm{~km}$ below the ionosphere. Figure 9 shows the satellite concept to obtain both HF wave amplitudes for signals scattered from the ocean and plasma parameters from the bottomside ionosphere at the satellite altitude.

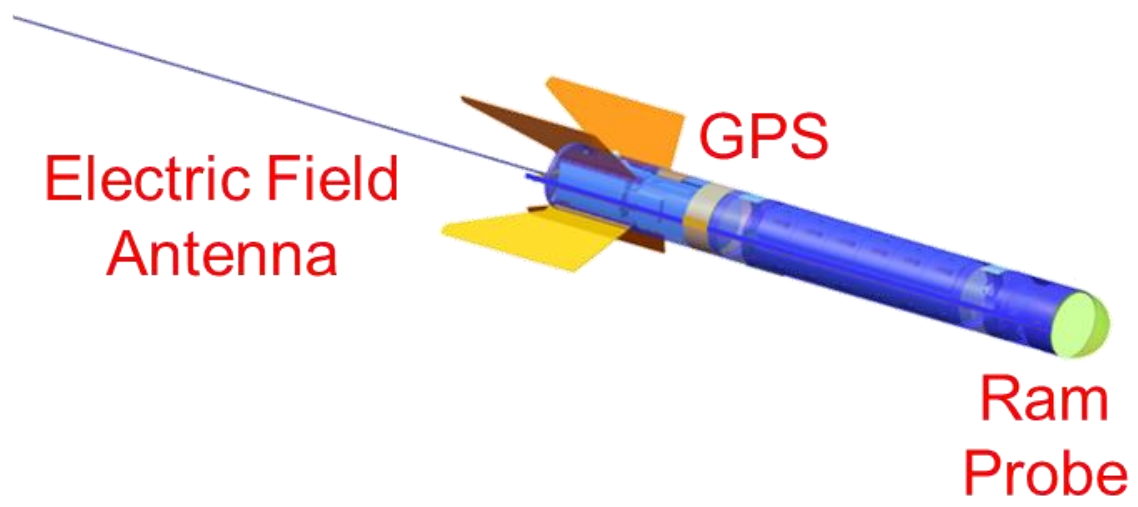

Figure 9. The CARINA satellite for flights below the ionosphere to measure HF radio scatter from the ocean along with in situ plasma properties. The Electric Field Antenna is connected to a radio receiver to form an electric dipole instrument in orbit. The Ram Probe measures the electron or ion density at the satellite orbit. The GPS receiver provides both satellite position and total electron content in the ionosphere above the satellite.

\section{Summary}

The GIOS program has conducted HF scatter and ionosphere propagation tests to show that a single HF transmitter can illuminate greater than $1000 \mathrm{~km}$ of ocean surface and scatter waves to single satellite in low earth orbit. The receiver in orbit samples the HF illuminated area to measure Brag scatter from the ocean with signals that pass through the ionosphere. The Doppler shifts and group delays map to specific points on the ocean surface at each point in the satellite orbit. Using the theory of ocean scatter, both coherent (specular) and incoherent (Bragg) scatter components at HF frequency provides bistatic sampling of global ocean surface. Data based models of the waveheight spectrum have been used in simulations to validate this concept. Experimental tests with the HF transmitter of ROTHR/VA and space based receiver of ePOP/RRI have provided data that were collected in April 2015. Actual interpretation in of the satellite measurements in terms of ocean surface parameters is in progress. A practical GIOS system will require measurements from a spacecraft flying below 
the ionosphere. For this purpose, the Naval Research Laboratory has designed a satellite called CARINA which will orbit the earth at an altitude of 200 to $250 \mathrm{~km}$ for 60 days.

Acknowledgements: We would like to thank co-investigators and the CASIOPE support team at University of Calgary. This work was supported by the 6.1 Base Program at the Naval Research Laboratory and the Defense Advanced Research Projects Agency (DARPA). The views, opinions, and/or findings expressed are those of the author and should not be interpreted as representing the official views or policies of the Department of Defense or the U.S. Government.

[1] Martin, S., An Introduction to Ocean Remote Sensing, Cambridge University Press, 2014.

[2] Barrick, D. E., "Remote sensing of sea state by radar" in Remote Sensing of the Troposphere, V. E. Derr, ed., Chapter 12, 1972

[3] S. R. Massel, Ocean Surface Waves: Their Physics and Prediction, World Scientific, New Jersey, 1996.

[4] G.T. Ruck, D.E. Barrick, W.D. Stuart, C.K. Krichbaum, Radar Cross Section Handbook, Volume 2, Plenum Press, New York, 1970.

[5] P. Beckmann, A. Spizzichino, The Scattering of Electromagnetic Waves from Rough Surfaces, MacMillan Co, New York, 1963.

[6] D.E. Barrick, W.H. Peake, A Review of Scattering From Surfaces With Different Roughness Scales, 3, pp. 865-868, 1968.

[7] James H. G., King E.P., White A., Hum R.H., Lunscher W.H.H.L., Siefring C.L. (2015), The ePOP Radio Receiver Instrument on CASSIOPE, Space Science Reviews, DOI: 10.1007/s11214-014-130-y. 